\title{
Evaluating Appropriateness of Prescribing of Long-Acting Risperidone for Injection in Acute Care Settings
}

\author{
Greg T Mah, Jane Dumontet, Anisha Lakhani, and Susan Corrigan
}

\begin{abstract}
Background: Long-acting risperidone for injection is a secondgeneration antipsychotic indicated for the treatment of schizophrenia and related psychotic disorders. It is a relatively new agent with pharmacokinetic and dosing properties unlike those of conventional long-acting antipsychotic drugs administered by injection.

Objective: To determine the proportion of patients for whom longacting risperidone for injection was prescribed appropriately in acute care settings in the Fraser Health Authority of British Columbia, according to the following 4 criteria: approved indication for therapy, 2-week dosing intervals, dose increases no sooner than every 4 weeks, and initial overlap supplementation with another antipsychotic for at least 3 weeks. A variety of other variables, including documented approval under special authority from the provincial drug coverage program, length of hospital stay, initial dose of risperidone, and total number of doses, were assessed as secondary outcomes.
\end{abstract}

Methods: A chart review was conducted for all patients for whom therapy with long-acting risperidone for injection was prescribed during stays in 8 acute care hospitals between July 1, 2007, and July 22, 2008. The appropriateness of prescribing was assessed according to the 4 prespecified criteria.

Results: Long-acting risperidone for injection was prescribed for 116 patients during the study period, and 82 of these started therapy and were included in the evaluation. The primary outcome could not be assessed for 27 of these 82 patients, because they were discharged early, and data for some or all of the 4 criteria were not available. For 33 (60\%) of the 55 remaining patients, long-acting risperidone for injection had been prescribed appropriately. In contrast, for $22(40 \%)$ of the patients, prescription of risperidone was deemed inappropriate because of failure to meet at least 1 of the 4 criteria. Premature escalation of the dose and inadequate overlap with antipsychotic supplementation were the most common reasons for designation of the prescription as inappropriate.

Conclusions: Opportunities exist to improve prescribing practices for long-acting risperidone for injection in acute care institutions in this health authority.

Key words: risperidone, antipsychotic agents, long-acting medications, delayed-action preparations, retrospective study, physicians' practice patterns

\section{RÉSUMÉ}

Contexte : La rispéridone injectable à action prolongée est un antipsychotique de deuxième génération indiqué pour le traitement de la schizophrénie et des troubles psychotiques associés. Il s'agit d'un agent relativement nouveau ayant un comportement pharmacocinétique et des caractéristiques posologiques différentes des antipsychotiques classiques injectables à action prolongée.

Objectif : Déterminer la proportion de patients à qui l'on a prescrit de la rispéridone injectable à action prolongée de façon appropriée en milieu de soins de courte durée à la Fraser Health Authority en Colombie-Britannique, sur la base des quatre principaux paramètres d'évaluation suivants : indication approuvée dans cette maladie, intervalles posologiques de deux semaines, augmentation de la dose à des intervalles d'au moins quatre semaines et administration concomitante initiale d'un autre antipsychotique pendant au moins trois semaines. Les paramètres d'évaluation secondaires comprenaient notamment l'approbation consignée et accordée en vertu de droits spéciaux par le régime provincial d'assurance-médicaments, la durée de l'hospitalisation, la dose initiale de rispéridone et le nombre total de doses administrées.

Méthodes : Une analyse des dossiers médicaux de tous les patients à qui la rispéridone injectable à action prolongée a été prescrite durant leur hospitalisation dans huit hôpitaux de soins de courte durée entre le $1^{\text {er }}$ juillet 2007 et le 22 juillet 2008 a été effectuée. La pertinence de la prescription du médicament a été évaluée en fonction des quatre critères prédéfinis.

Résultats : Un total de 116 patients ont reçu une prescription de rispéridone injectable à action prolongée durant la période de l'étude, et 82 de ces patients ont commencé le traitement et ont fait l'objet de l'évaluation. De ces 82 patients, 27 n'ont pas pu être évalués pour ce qui est des principaux paramètres d'évaluation, car ils ont reçu leur congé tôt et les données pour certains ou pour les quatre principaux paramètres d'évaluation n'étaient pas disponibles. Chez 33 (60\%) des 55 patients restants, la rispéridone injectable à action prolongée a été prescrite de façon appropriée. Par contraste, chez $22(40 \%)$ de ces patients, la prescription de rispéridone a été jugée inappropriée, car elle ne satisfaisait pas à au moins un des quatre critères. L'augmentation prématurée de la dose et l'administration concomitante inadéquate d'un autre antipsychotique étaient les raisons les plus courantes pour juger la prescription inappropriée.

Conclusions : Il existe des occasions d'améliorer les habitudes de prescription de la rispéridone injectable à action prolongée dans les établissements de soins de courte durée dans cette régie de la santé. 
Can J Hosp Pharm 2010;63(6):429-436
Mots clés : rispéridone, antipsychotiques, médicaments à action prolongée, préparations retard, étude rétrospective, habitudes de pratique des médecins

[Traduction par l'éditeur]

\section{INTRODUCTION}

Schizophrenia is a chronic psychiatric disorder that often $S_{\text {necessitates lifelong medical treatment. Patients with this }}$ condition may exhibit poor compliance with oral antipsychotic therapy. It has been reported that only one-third of such patients are fully adherent, taking their antipsychotics as prescribed, one-third are partially adherent, and the remaining third are completely nonadherent. ${ }^{1}$ Factors potentially contributing to noncompliance include the patient's lack of insight about the disease itself, drug-related adverse effects, lack of therapeutic rapport with medical professionals, misunderstandings about pharmacotherapy, and complex dosing schedules. ${ }^{1}$

Long-acting risperidone for injection was the first atypical antipsychotic available for depot injection in Canada., Its main advantage is improvement in compliance with therapy for patients who do not take their oral medications consistently. Also, as an atypical antipsychotic, risperidone has a lower risk of dose-dependent extrapyramidal movement disorders than conventional agents such as haloperidol. ${ }^{4,5}$

Long-acting risperidone for injection has a specially formulated drug delivery system, whereby particles of the drug are encapsulated into organic polymer matrixes called microspheres. ${ }^{6}$ These microspheres do not degrade appreciably, to release the risperidone, until 3 weeks after the first intramuscular injection. Accordingly, the manufacturer recommends that all patients receiving the drug for the first time should also receive overlapping therapy with another oral antipsychotic to maintain therapeutic plasma concentrations during the initial period. ${ }^{7}$ After the first dose, long-acting risperidone for injection is administered every 2 weeks to maintain oscillations between peak and trough levels within the therapeutic range. Peak levels of the drug are expected when most of the drug has been released from the microspheres, at 4 to 6 weeks after each injection. ${ }^{6}$ Steady-state concentrations in the plasma are reached after 4 injections. ${ }^{6}$ Given the unique pharmacokinetic profile of this drug, the manufacturer recommends that dose escalations be made no more frequently than every 4 weeks. ${ }^{7}$ Some experts have even suggested that the timeframe for assessing any specific dose of the drug be extended to 6 to 8 weeks after the initial dose to ensure that the patient's response is being observed at steady-state plasma concentrations. ${ }^{8}$

The unique pharmacokinetic characteristics of long-acting risperidone for injection may pose challenges to clinicians who are more familiar with conventional antipsychotics for depot injection, such as decanoate formulations of haloperidol, fluphenazine, flupenthixol, and zuclopenthixol.9 An understanding of and adherence to guidelines for appropriate prescribing may improve patient response, minimize doserelated side effects, and decrease unnecessary costs. An evaluation of prescribing and usage patterns for long-acting risperidone for injection within the Fraser Health Authority was undertaken to determine if patients were receiving this medication appropriately and if there was a need to provide education or create usage protocols to promote safe and effective drug therapy.

\section{METHODS}

This retrospective drug-use evaluation was conducted at 8 of the acute care hospitals of the Fraser Health Authority in British Columbia. In total, these 8 hospitals have about 2500 acute care beds. The study protocol was approved by the Fraser Health Research Ethics Board.

The primary outcome was the proportion of patients with a prescription for long-acting risperidone for injection for whom the drug was prescribed appropriately, according to the following 4 criteria: patient had an approved indication for therapy, dosing interval was appropriate, dose-escalation schedule was appropriate, and patient received antipsychotic supplementation (Table 1). The first criterion (approved indication for therapy) was based on the manufacturer's product monograph for long-acting risperidone for injection ${ }^{7}$ and the special authority requirements of British Columbia's drug coverage program, PharmaCare. The PharmaCare special authority program assesses patients' situations on a case-by-case basis, and grants full benefit status for medications dispensed in the community if they are prescribed for specific clinical indications. The provincial requirements were included because it would be problematic to discharge a patient with limited 
This single copy is for your personal, non-commercial use only.

For permission to reprint multiple copies or to order presentation-ready copies for distribution, contact CJHP at cjhpedit@cshp.ca

\section{Table 1. Criteria for Appropriate Prescribing of Long-Acting Risperidone for Injection*}

\begin{tabular}{|c|c|}
\hline Criterion & Details \\
\hline Indicationt & Schizophrenia or related psychotic disorder \\
\hline Dosing intervalł & 12-16 days after previous injection \\
\hline Dose escalation schedule $\neq$ & Not more frequently than every $25-28$ days \\
\hline Antipsychotic supplementation $\neq$ & $\begin{array}{l}\text { Oral antipsychotic supplementation for at least 18-21 days after first injection } \\
\text { OR } \\
\text { Conventional long-acting antipsychotic by injection within } 5 \text { days before } \\
\text { receiving first injection of risperidone }\end{array}$ \\
\hline \multicolumn{2}{|c|}{$\begin{array}{l}\text { *Achievement of all } 4 \text { criteria was required for designation as "appropriate prescribing". } \\
\text { †Based on manufacturer's product monograph7 for long-acting risperidone by injection and special authority } \\
\text { requirements of the British Columbia drug coverage program (PharmaCare). After the study was completed, } \\
\text { long-acting risperidone for injection was also approved in Canada for the treatment of bipolar I disorder. } \\
\text { fFor the dosing interval, dose escalation schedule, and antipsychotic supplementation criteria, a grace interval was } \\
\text { predefined to allow for the fact that transcription of a prescription to the electronic medical record does not always } \\
\text { occur on the same date as the physician writes the order. Selection of these criteria was based on the dosing } \\
\text { recommendations in the manufacturer's product monograph.? }\end{array}$} \\
\hline
\end{tabular}

financial resources with a prescription for a relatively expensive medication, such as long-acting risperidone for injection, without ensuring that he or she would have adequate financial support in the community. The remaining 3 criteria for appropriate prescribing were based on dosing recommendations in the product monograph. ${ }^{7}$ Achievement of all 4 criteria was required for designation of prescribing as appropriate.

The following secondary outcomes were also determined: proportion of patients with documented predischarge approval from PharmaCare for special authority drug coverage for risperidone, length of the hospital stay, number of doses of risperidone received during the admission, initial dose of risperidone received in hospital, and number of antipsychotic medications prescribed before admission and at the time of discharge from hospital. The investigators also wanted to determine if there was an overall reduction in the use of oral antipsychotics, as long-acting risperidone for injection is expected to reduce the number of medications taken orally, following appropriate dosage titration.

Patients for whom long-acting risperidone for injection was prescribed between July 1, 2007, and July 22, 2008, as recorded in the computer system of each acute care hospital, were eligible for the study. Each patient's chart and electronic record were screened to determine if he or she met the study's eligibility criteria. Patients were included if they had been newly started on long-acting risperidone for injection during the index hospital admission. Patients were excluded if they had received this drug formulation at any time within the 6 weeks before the index admission. Patients were also excluded if there were insufficient data in their health records to allow full assessment of the criteria for appropriateness of prescribing. For example, if a patient was discharged soon after receiving his or her first dose and there were no medication-related discharge notes, it was not possible to determine if the dosing interval had been every 2 weeks, if dosage increases had been ordered no sooner than every 4 weeks, or if there was sufficient overlap with oral antipsychotic supplementation for at least 3 weeks.

A standardized data collection form was used to record relevant patient information. Descriptive statistics, including means and standard deviations for proportional prevalence data, are reported here. After the results were compiled and analyzed, the study investigators planned to meet with stakeholders from physician, pharmacy, and nursing groups to discuss how prescribing of this agent might be improved and to promote appropriateness of use, if needed.

\section{RESULTS}

A total of 116 patients for whom long-acting risperidone for injection was prescribed at 1 of the 8 acute care sites over the study period were identified and screened (Figure 1). For 82 (71\%) of these, risperidone therapy had been initiated during the index admission, and they were screened further for inclusion in the study. The remaining 34 (29\%) patients were excluded either because they had not received at least 1 dose of the drug or because they were continuing therapy that had been initiated in the community. Of the 82 patients who were newly started on long-acting risperidone for injection, 27 were excluded because of early discharge from hospital, most within 2 weeks after receiving their first dose. For these 27 patients, there were insufficient data to assess the appropriateness of dosing interval, the dose escalation schedule, or the provision of antipsychotic supplementation. Therefore, 55 patients were included in the study (Table 2).

The proportion of patients for whom the individual appropriateness criteria were fulfilled varied from $78 \%$ to $96 \%$ (Figure 2). For $33(60 \%)$ of the 55 patients included in the evaluation, all 4 criteria were fulfilled and prescribing was deemed appropriate. Considering each criterion individually, 2 patients (4\%) had an inappropriate indication for the drug (bipolar I disorder without psychotic features), 5 (9\%) received 
Patients with prescription for long-acting risperidone for injection $(n=116)$

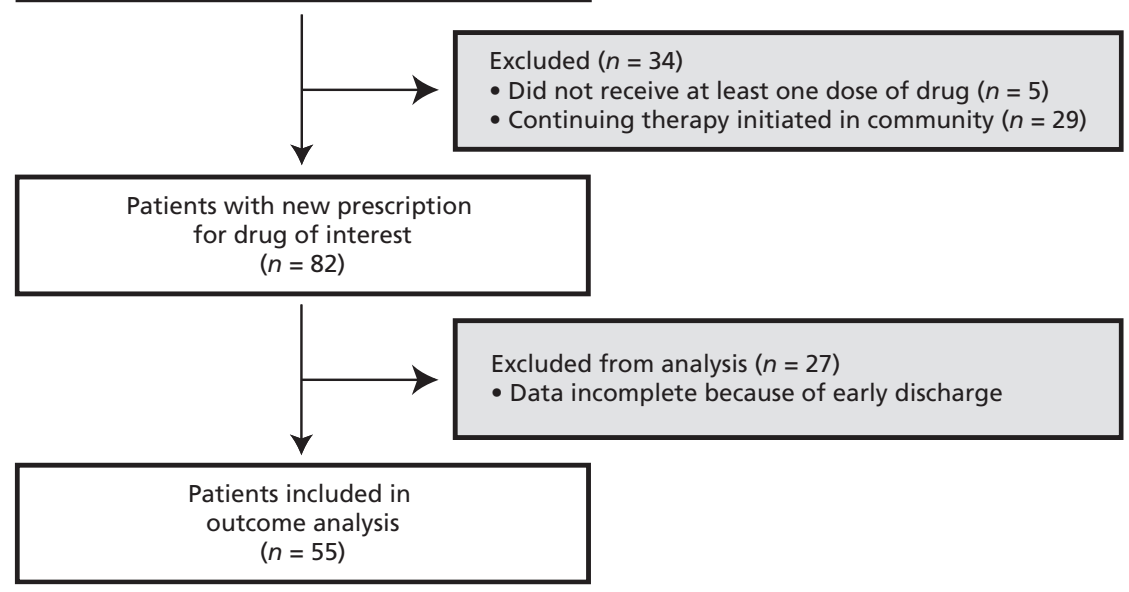

Figure 1. Selection of patients for inclusion in an evaluation of appropriateness of prescribing of long-acting risperidone for injection.

Table 2. Baseline Characteristics of 55 Patients Included in Evaluation of Appropriateness of Prescribing of Long-Acting Risperidone for Injection

Characteristic No. (\%) of Patients* $(n=55)$

\section{Demographic}

Age, mean \pm SD $(y r)$

$40 \pm 14$

Sex (males)

$29 \quad(53)$

\section{Factors contributing to admission}

Decompensation

54 (98)

Noncompliance with therapy

$44 \quad(80)$

Trial of long-acting risperidone for injection

2 (4)

Prescriber of long-acting risperidone for injection

Psychiatrist

Other physician

$0 \quad(0)$

\section{Primary psychiatric (axis I) diagnosis}

Schizophrenia

Schizoaffective disorder

Psychosis not otherwise specified

Bipolar disorder (with or without psychotic features)

$6 \quad(11)$

\section{Residence before hospital admission}

Independent living

Family home

Boarding home or semi-independent living

Other hospital

No fixed address

Hotel or motel

$1 \quad(2)$

$\mathrm{SD}=$ standard deviation

*Except where indicated otherwise. 


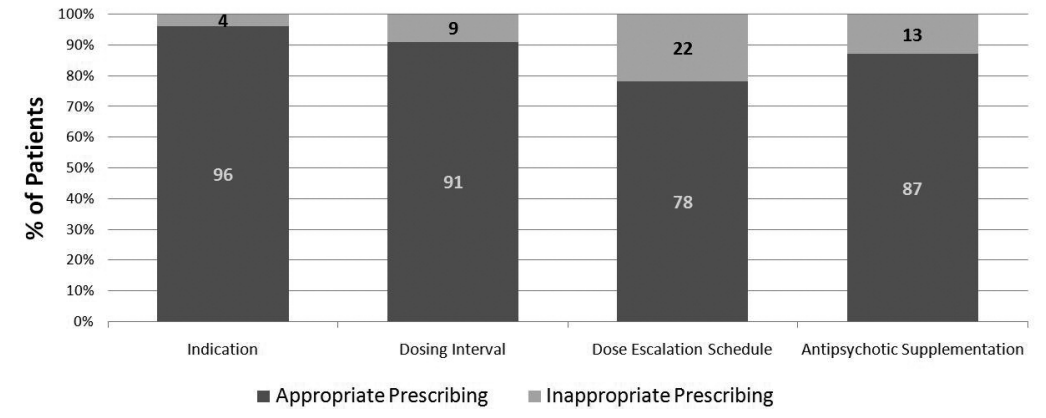

Figure 2. Proportion of 55 patients newly started on long-acting risperidone for injection for whom individual appropriateness criteria were met.

the second dose of risperidone by injection too early or too late, and for $12(22 \%)$ the dose was escalated too quickly (earlier than every 4 weeks). Of the 12 patients for whom dosing escalation was inappropriate, 9 received an initial dose of $25 \mathrm{mg}$ and a second dose of $37.5 \mathrm{mg} 2$ weeks later. Seven (13\%) of the patients did not receive adequate antipsychotic supplementation to overlap with the initiation of long-acting risperidone for injection. Of these patients, one did not receive any supplemental therapy. For the remainder, oral antipsychotic supplementation was prescribed but for less than the recommended minimum duration of 3 weeks. For 4 (7\%) of the 55 patients, prescribing was deemed inappropriate because of failure to fulfill multiple criteria.

Only 28 (51\%) of the patients had documented predischarge approval under special authority from PharmaCare for long-acting risperidone therapy. Other secondary outcomes are reported in Table 3.

\section{DISCUSSION}

This retrospective evaluation focused on patients newly started on long-acting risperidone for injection because the appropriateness of this agent during the initial months of therapy requires an understanding of its unique pharmacokinetic characteristics. Thus, we aimed to describe the prescribing patterns for this relatively new antipsychotic formulation and to identify any knowledge gaps. The results showed that for $40 \%$ of the patients for whom appropriateness could be assessed, one or more of the criteria for appropriate prescribing were not fulfilled. This indicates a significant opportunity within the Fraser Health Authority to improve the use of this agent and to optimize patient outcomes.

In this study we were unable to assess overall appropriateness of prescribing for 27 (33\%) of the 82 patients newly started on long-acting risperidone, largely because of early discharge from hospital. If it is assumed that prescribing was appropriate for all of these patients, then the overall proportion of patients with appropriate initial therapy would have been $73 \%$ (60/82). Conversely, if it is assumed that prescribing was inappropriate for all of these patients, then the overall proportion would have been only $40 \%$ (33/82). The actual rate of appropriate prescribing probably lies between these 2 values.

At the time of this study's design, in June 2008, the only indications for long-acting risperidone for injection approved by Health Canada were schizophrenia and related psychotic disorders. Two of the patients in this study, who had bipolar I disorder, did not meet the study criterion for indication, but the drug has since been approved in Canada for this condition. ${ }^{7}$ As such, no improvements in prescribing are required in this area. However, the inclusion of bipolar I disorder as an indication for special authority coverage of longacting risperidone for injection should be reassessed by PharmaCare.

In this study, 5 (9\%) patients received the drug at intervals other than every 2 weeks. Among those patients, orders for a single dose were common, with no specification that the dose should be repeated at 2 -week intervals. As a result, subsequent doses were given either 1 week early or 1 week late. Unlike the situation for other long-acting drugs given by injection, the effect of the first dose of long-acting risperidone will not be evident at the time the second dose is due; therefore, there is no pharmacokinetic reason to wait to specify the frequency or dose for the second injection. It is also important that orders for long-acting risperidone be written as continuation orders, to be administered every 2 weeks, so that the medication will be included on the active medication list when the patient is transferred or discharged from the hospital.

A majority of patients newly started on long-acting risperidone received an initial dose of $25 \mathrm{mg}$. This is consistent with current recommendations for dose initiation, ${ }^{7}$ based on a prospective fixed-dose study that showed no additional benefit of starting with dosages above $25 \mathrm{mg}$ every 2 weeks. ${ }^{2}$ In a more recent prospective study, however, in which patients receiving long-acting risperidone by injection were followed for 3 years, 
Table 3. Secondary Outcomes

\begin{tabular}{lc} 
Outcome & $\begin{array}{c}\text { No. (\%) of Patients } \\
\text { or Mean } \pm \text { SD }(\boldsymbol{n}=\mathbf{5 5})\end{array}$ \\
\hline $\begin{array}{l}\text { Documented approval under special authority of } \\
\text { provincial drug coverage program }\end{array}$ & $28(51)$ \\
\hline Length of hospital stay (days) & $39 \pm 24$ \\
Since admission & $30 \pm 21$ \\
Since first dose of long-acting risperidone by injection & $2.6 \pm 1.2$ \\
\hline No. of doses of long-acting risperidone by injection & $3(5)$ \\
received in hospital & $49(89)$ \\
Initial dose of long-acting risperidone by injection for new starts & $3(5)$ \\
$12.5 \mathrm{mg}$ & $0(0)$ \\
$25 \mathrm{mg}$ & $1.1 \pm 0.7$ \\
$37.5 \mathrm{mg}$ & $1.7 \pm 0.6$ \\
$50 \mathrm{mg}$ & \\
\hline No. of regular antipsychotics & \\
Before admission & \\
At discharge & \\
SD = standard deviation. &
\end{tabular}

the authors found that patients were more likely to continue with therapy if the dosage was higher than $25 \mathrm{mg}$ every 2 weeks. ${ }^{10}$ Given that current evidence pertaining to the dose-response relation for long-acting risperidone by injection is unclear, the current study did not include the initial dose as a criterion for evaluating the appropriateness of prescribing.

Three patients in the current study started long-acting risperidone at a dose of $12.5 \mathrm{mg}$. Despite a lack of clinical trials evaluating this dose, the manufacturer suggests that a starting dose of $12.5 \mathrm{mg}$ may be appropriate for patients with certain clinical factors, such as renal or hepatic impairment, drug interactions with inhibitors of the cytochrome P450 2D6 isoenzyme (which may increase plasma levels of risperidone), or a history of movement disorders with antipsychotics. ${ }^{7}$ According to the results of serum creatinine and liver enzyme tests, none of the patients who were started on the $12.5-\mathrm{mg}$ dose had clinically significant renal or hepatic dysfunction, nor were any of them taking other medications that would have interacted significantly with risperidone. In addition, all of these patients received $25 \mathrm{mg}$ as their second dose. Therefore, the reasons for starting these 3 patients on a lower dose of long-acting risperidone were not apparent and were unlikely to have been clinically meaningful.

Dose escalations of long-acting risperidone should not be made more frequently than every 4 weeks, given that observable drug effects should not be anticipated before this time. In an academic detailing study carried out in the United Kingdom, Paton and others ${ }^{11}$ found that only $50 \%$ of prescribing psychiatrists understood that it takes at least 6 weeks to reach steady-state plasma concentrations at any given dose. In the present study, almost one-quarter of the patients received premature dose increases. Most of these patients received an initial 25-mg dose, followed by a 37.5-mg dose 2 weeks later. However, according to information available in the health records, there was no evidence to suggest that any of these patients were stabilized and that they had tolerated higher doses of long-acting risperidone in the past. The most common reason for increasing the dose of the second injection was lack of efficacy. Given the kinetics of long-acting risperidone for injection, whereby appreciable drug release does not occur until 3 weeks after the first injection, this practice would be questionable, because patients and clinicians would not have had an opportunity to assess any response at 2 weeks after the initial injection. Clinicians should also be aware that if the dose is increased too quickly, it becomes more difficult to manage possible extrapyramidal side effects, since the medication will remain in the patient's system for a prolonged period. Additionally, if a higher dose is the ultimate target, then starting at $25 \mathrm{mg}$ simply prolongs the time to achieve steady state. To see the effects of any specific dose, conservative practitioners should wait at least 6 weeks to assess patients at steady-state drug levels before making dose adjustments. This appears to represent a significant gap in prescribers' knowledge about this drug formulation.

Long-acting risperidone for injection is the only antipsychotic for depot injection currently available for which antipsychotic supplementation for at least 3 weeks is recommended for new users. ${ }^{12}$ Prescribers should be aware that the drug has a significantly slower onset of action than conventional antipsychotic depot drugs. ${ }^{13}$ In this study, $13 \%$ of patients did not receive 3 full weeks of antipsychotic supplementation. This is similar to the finding in a larger retrospective study that $16.5 \%$ of 1232 acute care patients did not receive oral supplementation during the first 3 weeks. ${ }^{14}$ 
Practitioners who do not prescribe concurrent antipsychotic therapy for at least 3 weeks after starting patients on longacting risperidone by injection should not anticipate any clinical benefits to be derived from the long-acting risperidone during this initial period, since there will be no appreciable level of the drug in the systemic circulation.

The results of this study were presented to various stakeholders to encourage discussion and implementation of improvement strategies. As a result, an education strategy was implemented within the Fraser Health Authority to facilitate appropriate prescribing of long-acting risperidone by injection. Education sessions for physicians, pharmacists, and nurses were conducted at the acute care sites to highlight the pharmacokinetic aspects of long-acting risperidone by injection related to dose escalation strategies and the requirement for overlapping antipsychotic supplementation during initial therapy. Also, a preprinted physician order form was introduced (Appendix 1; see www.cjhp-online.ca/index.php/cjhp/issue/view/78), containing guidance about indications for this drug formulation, criteria for provincial drug coverage, and dosing recommendations; the form also includes a section for ordering antipsychotic overlap supplementation for patients who are starting therapy with long-acting risperidone. This form, which highlights the drug's complex dosing and pharmacokinetic characteristics, must now be completed for new patients starting therapy with longacting risperidone before the drug is dispensed at any Fraser Health acute care site.

Long-acting risperidone for injection is considerably more expensive than generic risperidone products for oral administration and other currently available conventional long-acting antipsychotics for injection, with the 25-mg and 50-mg doses costing about $\$ 150$ and $\$ 300$ per injection, respectively (as of summer 2010). Therefore, it is expected to have a significant impact on the drug budget of the Fraser Health Authority. In British Columbia, outpatients require PharmaCare special authority approval if they need the provincial government to cover the costs of long-acting risperidone for injection. The high cost may be a barrier to compliance for psychiatric patients without adequate public or private drug coverage once they are discharged from hospital. During data collection for the current study, it was noted that documentation of PharmaCare special authority approval for outpatient drug coverage was inconsistent and often missing from patient charts. Specifically, special authority approval was documented in the health records for only $51 \%$ of patients newly started on long-acting risperidone for injection. For patients who cannot afford the drug, this lack of documentation might lead to treatment interruption after discharge, as extra communication among the receiving psychiatrist, the community pharmacy, and the provincial drug coverage program would be needed before patients could receive the next injection. In the current study, it is possible that special authority approval was arranged by the prescribing physician via telephone, without documentation in the chart, but there was no mechanism for us to capture cases in which this occurred. A standardized method of documenting special authority approval (i.e., the aforementioned preprinted order), accessible to all individuals involved with patient care, including psychiatrists, nurses, pharmacists, social workers, and case managers, would probably help to facilitate seamless transition from acute care settings to community environments by ensuring that patients do not miss their injections because of cost.

On average, patients received fewer than 3 injections of long-acting risperidone and stayed in the inpatient psychiatric unit for 30 days after their first injection. These findings corroborate results reported by De la Gandara and others who found that patients in their retrospective study received an average of 2 injections over a mean hospital stay of 25 days. ${ }^{14}$ Since appreciable release of long-acting risperidone should not be anticipated any earlier than 3 weeks after the first injection and since steady-state drug levels are not reached until week 6 , our findings suggest that the patients in acute care settings were not necessarily stabilized because of the introduction of the risperidone, but rather because they were treated in a structured and supportive care environment with concurrent oral antipsychotic therapy. It is likely that the effects of the long-acting risperidone did not begin to appear until just before discharge, if at all. One of the limitations of this study is that it was not designed to follow use of antipsychotic medications or patient outcomes after discharge.

One of the main reasons for the introduction of longacting risperidone for injection was to improve compliance with medication therapy by simplifying dosing regimens. More specifically, the responsibility for administering a medication is shifted from patients, who would be expected to take one or more oral antipsychotics faithfully on a daily basis, to clinicians, who administer the long-acting formulation every 2 weeks. For that reason, it is expected that patients started on long-acting risperidone by injection would eventually discontinue use of oral antipsychotics. Interestingly, in this study the average number of regularly scheduled antipsychotics was 1.1 before hospital admission and 1.7 at the time of discharge. Thus, the average number of antipsychotics increased after initiation of the long-acting risperidone. This finding can be explained by the fact that most patients did not remain in the hospital long enough for complete tapering of their oral antipsychotics. Stabilization on long-acting risperidone might be expected several weeks after initiation of therapy. Hence, a long-term study in an outpatient setting is required to determine if there is an overall reduction in the total number of antipsychotic medications as a result of therapy with long-acting risperidone by injection. 
This study was limited by its retrospective nature, the lack of follow-up with patients after discharge from hospital, and the lack of information about clinical outcomes achieved when therapy with long-acting risperidone was started. However, the purpose of the study was to describe current prescribing patterns at the acute care sites of one health authority and to find ways to improve these patterns. Studies evaluating the clinical efficacy and safety of this drug formulation have been published elsewhere. ${ }^{2,3,10,15}$ Future studies with follow-up of patients in community settings after hospital discharge would help to describe prescribing patterns such as dosing changes, tapering of oral antipsychotics, and total duration of therapy with long-acting risperidone.

\section{CONCLUSIONS}

The results of this study indicate that the current prescribing patterns for long-acting risperidone for injection within the acute care settings of the Fraser Health Authority could be improved to meet current recommendations, which are based on the pharmacokinetics of the drug. Methods to improve prescribing patterns may include multidisciplinary education sessions (for physicians, pharmacists, and nurses) and implementation of a regional preprinted physician order form with dosing guidelines and strategies to ensure seamless care during transition from acute care to community settings. Further study is needed to determine whether these interventions will affect the appropriateness of prescribing of this drug in acute care sites.

\section{References}

1. Burton SC. Strategies for improving adherence to second-generation antipsychotics in patients with schizophrenia by increasing ease of use. $J$ Psychiatr Pract 2005;11(6):369-378.

2. Kane JM, Eerdekens M, Lindenmayer JP, Keith SJ, Lesem M, Karcher K. Long-acting injectable risperidone: efficacy and safety of the first longacting atypical antipsychotic. Am J Psychiatry 2003;160(6):1125-1132.

3. Chue P, Eerdekens M, Augustyns I, Lachaux B, Molcan P, Eriksson L, et al. Comparative efficacy and safety of long-acting risperidone and risperidone oral tablets. Eur Neuropsychopharmacol 2005;15(1):111-7.

4. Moller HJ. Long-acting injectable risperidone for the treatment of schizophrenia—clinical perspectives. Drugs 2007;67(11):1541-1546.

5. Hunter R, Kennedy E, Song F, Gadon L, Irving CB. Risperidone versus typical antipsychotic medication for schizophrenia. Cochrane Database Syst Rev 2003;(2):CD000440.

6. Wilson WH. A visual guide to expected blood levels of long-acting injectable risperidone in clinical practice. J Psychiatr Pract 2004;10(6): 393-401.

7. Risperdal Consta: risperidone powder for injectable prolonged-release suspension $12.5 \mathrm{mg}, 25 \mathrm{mg}, 37.5 \mathrm{mg}$ and $50 \mathrm{mg}$ antipsychotic agent [product monograph]. Toronto (ON): Janssen-Ortho Inc; 2009 [cited 2010 Nov 1]. Available from: http://webprod.hc-sc.gc.ca/dpd-bdpp/ info.do?lang=eng\&code=74089
8. Marder SR, Conley R, Ereshefsky L, Kane JM, Turner MS. Clinical guidelines: dosing and switching strategies for long-acting risperidone. J Clin Psychiatry 2003;64 Suppl 16:41-46.

9. Kane JM, Aguglia E, Altamura AC, Ayuso Gutierrez JL, Brunello N, Fleischhacker WW, et al. Guidelines for depot antipsychotic treatment in schizophrenia. Eur Neuropsychopharmacol 1998;8(1):55-66.

10. Taylor DM, Fischetti, C, Sparshatt, A, Thomas A, Bischara D, Cornelius V. Risperidone long-acting injection: a prospective 3-year analysis of its use in clinical practice. J Clin Psychiatry 2009;70(2):196-200.

11. Paton C, Adebowale O, Okocha CI. The use of academic detailing to improve evidence based prescribing of risperidone long acting injection. Int J Psychiatry Clin Pract 2008;12(3):210-214.

12. Rainer MK. Risperidone long-acting injection: a review of its long term safety and efficacy. Neuropsychiatr Dis Treat 2008;4(5):919-927.

13. Altamura AC, Sassella F, Santini A, Montresor C, Fumagalli S, Mundo E. Intramuscular preparations of antipsychotics-uses and relevance in clinical practice. Drugs 2003;63(5):493-512.

14. De la Gandara J, Molina LS, Rubio G, Rodriguez-Morales A, Borrajo $\mathrm{RH}$, Buron JA. Experience with injectable long-acting risperidone in long-term therapy after an acute episode of schizophrenia: the SPHERE study. Expert Rev Neurother 2009;9(10):1463-1474.

15. Fleishhacker WW, Eerdekens, M, Karcher, K, Remington, G, Llorca, PM, Chrzanowski, W, et al. Treatment of schizophrenia with long-acting injectable risperidone: a 12-month open-label trial of the first longacting second-generation antipsychotic. J Clin Psychiatry 2003;64(10): $1250-1257$.

Greg T Mah, BScPharm, is a Clinical Pharmacist, Burnaby Hospital, Fraser Health, Burnaby, British Columbia.

Jane Dumontet, BScPharm, PharmD, is a Clinical Pharmacy Specialist, Cottonwood and Connolly Lodges, Fraser Health, Coquitlam, British Columbia.

Anisha Lakhani, BScPharm, PharmD, is the Pharmacotherapy Evaluation and Optimization Coordinator, Fraser Health, Surrey, British Columbia.

Susan Corrigan, BScPharm, PharmD, is a Clinical Pharmacy Practice Leader, Surrey Memorial Hospital, Fraser Health, Surrey, British Columbia.

\section{Address correspondence to:}

Greg T Mah

Department of Pharmacy

Burnaby Hospital

Fraser Health Authority

3935 Kincaid Street

Burnaby BC V5G 2X6

e-mail: Greg.Mah@fraserhealth.ca

\section{Acknowledgements}

We thank Dr Aaron Tejani, Clinical Research and Drug Information Coordinator with Fraser Health, for his support and guidance during the study. We also thank Michael Wasdell of the Department of Evaluation and Research Services, Fraser Health, for his input regarding the reporting of study results. Lastly, we thank Dr Paul Waraich, a psychiatrist with Fraser Health, for his involvement in developing Fraser Health's preprinted protocol for prescribing long-acting risperidone for injection. 